\title{
ARTICLE OPEN Tailored morphology and highly enhanced phonon transport in polymer fibers: a multiscale computational framework
}

\author{
Shangchao Lin $\mathbb{B}^{1,4 *}$, Zhuangli Cai ${ }^{1,2,4}$, Yang Wang ${ }^{2}$, Lingling Zhao ${ }^{2}$ and Chenxi Zhai $\mathbb{C}^{3}$
}

\begin{abstract}
Although tremendous efforts have been devoted to enhance thermal conductivity in polymer fibers, correlation between the thermal-drawing conditions and the resulting chain alignment, crystallinity, and phonon transport properties have remained obscure. Using a carefully trained coarse-grained force field, we systematically interrogate the thermal-drawing conditions of bulk polyethylene samples using large-scale molecular dynamics simulations. An optimal combination of moderate drawing temperature and strain rate is found to achieve highest degrees of chain alignment, crystallinity, and the resulting thermal conductivity. Such combination is rationalized by competing effects in viscoelastic relaxation and condensed to the Deborah number, a predictive metric for the thermal-drawing protocols, showing a delicate balance between stress localizations and chain diffusions. Upon tensile deformation, the thermal conductivity of amorphous polyethylene is enhanced to $80 \%$ of the theoretical limit, that is, its pure crystalline counterpart. An effective-medium-theory model, based on the serial-parallel heat conducting nature of semicrystalline polymers, is developed here to predict the impacts from both chain alignment and crystallinity on thermal conductivity. The enhancement in thermal conductivity is mainly attributed to the increases in the intrinsic phonon mean free path and the longitudinal group velocity. This work provides fundamental insights into the polymer thermal-drawing process and establishes a complete process-structure-property relationship for enhanced phonon transport in all-organic electronic devices and efficiency of polymeric heat dissipaters.
\end{abstract}

npj Computational Materials (2019)5:126; https://doi.org/10.1038/s41524-019-0264-2

\section{INTRODUCTION}

Polymer fibers have been widely explored in many engineering applications due to the advantages of their anti-corrosion, hightoughness, and low-density features. However, bulk polymers, either (semi)-crystalline or amorphous, exhibit low thermal conductivities (TCS) of only $0.1-0.3 \mathrm{~W} \mathrm{~m}^{-1} \mathrm{~K}^{-1}$ at room temperature. ${ }^{1}$ Such poor performance in heat conduction limits their applications in electronic packaging, ${ }^{2}$ aerospace materials, ${ }^{3}$ and automotive parts. ${ }^{4}$ The low TC in bulk polymers have been attributed to various fundamental mechanisms, including the disordered arrangement of polymer chains in the amorphous phase, the presence of grain boundaries between polycrystalline domains, inter-chain entanglements, nanovoids, and other defects and impurities, all of which result in significant phonon scatterings that hinder heat conduction. ${ }^{5-8}$ Nevertheless, fully extended (with all the dihedrals in the trans state) single polyethylene (PE) chain has been proved to possess extremely high TC in the order of $100 \mathrm{~W} \mathrm{~m}^{-1} \mathrm{~K}^{-1}$ through all-atomistic molecular dynamics (AA-MD) simulations. ${ }^{9}$ This highlights the potential of highly efficient heat conduction in bulk polymers, if all the chains are extended and aligned along the heat conducting direction.

Numerous experimental studies have shown that tensile elongation in the thermal-drawing process can effectively enhance the TC in bulk polymer fibers. Shen et al. ${ }^{10}$ fabricated high-quality ultra-drawn polyethylene (PE) nanofibers with roomtemperature TC up to $104 \mathrm{~W} \mathrm{~m}^{-1} \mathrm{~K}^{-1}$ at a draw ratio of 400 due to the restructuring of polymer chains towards alignment and crystallization. Single crystalline PE mats with a draw ratio of $\lambda=350$ were also found to exhibit excellent TC in the order of
$40 \mathrm{~W} \mathrm{~m}^{-1} \mathrm{~K}^{-1}$, attributed to the presence of $\sim 85 \%$ of long needleshaped crystals. ${ }^{11}$ Recently, room-temperature PE microfibers ${ }^{12}$ with a high TC of $51 \mathrm{~W} \mathrm{~m}^{-1} \mathrm{~K}^{-1}$, as well as PE nanofibers ${ }^{13}$ with both ultra-high strength of $11 \mathrm{GPa}$ and TC of around $50 \mathrm{~W} \mathrm{~m}^{-1} \mathrm{~K}^{-1}$ were reported. The current strategies to align individual PE chains and increase local crystallinity have been proven successful in enhancing the TC of polymeric materials; however, most studies rely on trial-and-error experimentations with few theoretical guidance. In particular, the optimization of the thermal-drawing protocols, including the annealing profile, draw ratio (tensile strain), and draw rate (strain rate), remains largely unexplored at the molecular level.

To complement experimental studies, AA-MD simulations have been widely applied to study chain alignment and heat conduction in many polymer systems. ${ }^{14-21}$ Using AA-MD simulations, Liu and Yang $^{22}$ show that the TC of bulk PE increases to $1.3 \mathrm{~W} \mathrm{~m}^{-1} \mathrm{~K}^{-1}$ at a strain of 2 , and interestingly, such enhancement in TC is more significant when the strain rate is smaller. They found that the alignment of polymer chains, measured by the Herman's orientational order parameter, also increases with strain, which correlates well with the increase in TC. By stretching amorphous polymer fibers at $500 \mathrm{~K}$, followed by quenching at $300 \mathrm{~K}$, Zhang and Luo ${ }^{23}$ achieved a 176-fold increase in TC at a strain of $\sim 9$ in AA-MD simulations. However, the TC vs. strain predicted that there does not reach a plateau as observed in experiments, ${ }^{11,24}$ but instead exhibits singularity at strains larger than 10 . This is probably due to the fact that the chains are covalently bonded across the periodic boundary of the simulation cell, which would undergo unphysical backbone stretching at high

\footnotetext{
${ }^{1}$ Institute of Engineering Thermophysics, School of Mechanical and Power Engineering, Shanghai Jiao Tong University, Shanghai 200240, China. ${ }^{2}$ Key Laboratory of Energy Thermal Conversion and Control of Ministry of Education, School of Energy and Environment, Southeast University, Nanjing, Jiangsu 210096, China. ${ }^{3}$ Department of Mechanical Engineering, FAMU-FSU College of Engineering, Florida State University, Tallahassee, FL 32310, USA. ${ }^{4}$ These authors contributed equally: Shangchao Lin, Zhuangli Cai. *email: shangchaolin@sjtu.edu.cn
} 
(a)

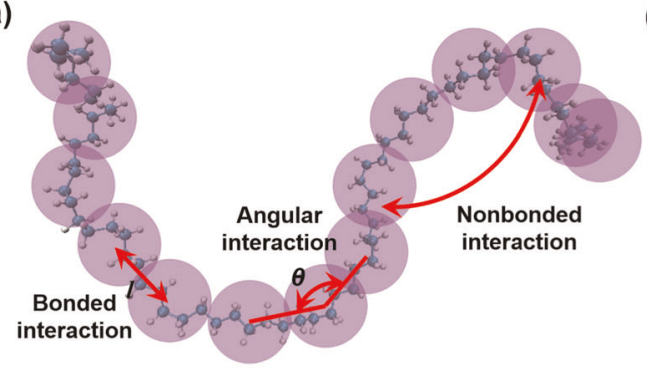

(c)

(b) $\varepsilon=0$

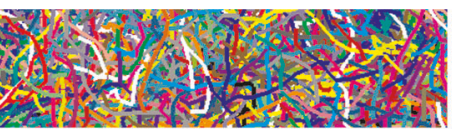

$\varepsilon=2$

$\varepsilon=10$

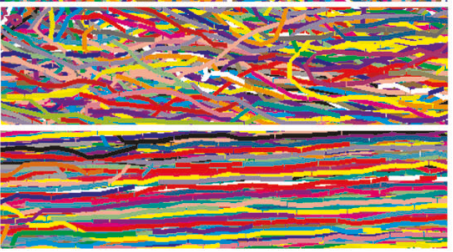

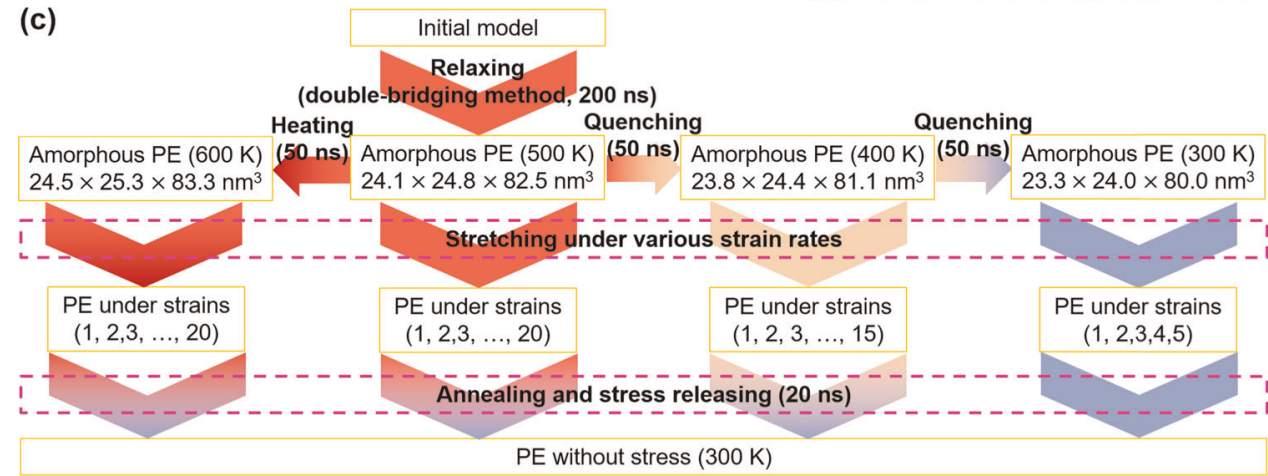

Fig. 1 Simulation model and procedure. a Schematic of the coarse-grained (CG) model for $\mathrm{PE}$, in which every five methylene groups (- $\mathrm{CH}_{2}-$ ) are represented by one bead. b CG-MD simulation snapshots of PE fibers thermally strained at $400 \mathrm{~K}$ under a strain rate of $5 \times 10^{6} \mathrm{~s}^{-1}$ under different strains after stress relaxation. c Flowchart of the simulated fiber thermal-drawing protocol with details discussed in Methods.

strains and lose the opportunity for inter-chain sliding or detachment to release the residue stress. Zhang and Luo $^{25}$ also investigated the morphology-dependent TC in amorphous PE by artificially tuning the chain stiffness. They found that the enhanced crystallinity and chain alignment along the heat conducting direction can lead to an increase in TC by more than one order of magnitude compared to amorphous PE. ${ }^{25}$

In spite of the above experimental and theoretical advances, the impacts from the fiber thermal-drawing protocols on polymer chain alignment, crystallinity, and the resulting phonon transport properties remain obscure. In particular, there are great interests for a scalable modeling framework to cover both thermal phonon transport properties of individual polymer chains at the nanoscale and morphological transitions during the thermal-drawing process at the mesoscale. For example, Liu and $\mathrm{Yang}^{22}$ simulated individual PE chains consisting of up to 400 carbon atoms, corresponding to a chain molecular weight of $5602 \mathrm{~g} \mathrm{~mol}^{-1}$, while the typical molecular weight of high-density PE is up to $10^{6} \mathrm{~g}$ mol $^{-1,26}$ which would have a critical influence on the TC of polymers. $^{27,28}$ From experiments ${ }^{29,30}$ and simulations, ${ }^{28}$ it is well known that longer polymer chains could lead to larger TCs, if the polymers are well aligned. This is probably due to the resulting longer thermal pathways through the chain, along which phonons can transport with less scattering and longer mean free path (MFP). ${ }^{31}$ In addition to the length scale above, the time scale involved in the fiber thermal-drawing process is also beyond the scope of conventional AA-MD simulations. Such time-scale limitation in AA-MD requires very high strain rates to be applied, which would inhibit relaxations of residue stresses in polymers. A scalable modeling framework, enabled by coarse-grained (CG) MD simulations, could greatly extend the length and time scales of conventional AA-MD simulations by two orders of magnitude. ${ }^{32-35}$ However, to the best of our knowledge, the application of CG-MD simulations to precisely model heat conduction in thermally drawn bulk polymer fibers has not been reported yet.

In this work we develop a predictive CG force field (Fig. 1a) for bulk PE (up to a relatively high molecular weight of $10,500 \mathrm{~g}$ $\mathrm{mol}^{-1}$ ), capable of reproducing the correct thermal transport and phase transition features. This is achieved through target property matching and the multistate-iterative Boltzmann inversion (MS-IBI) method $^{36}$ with our AA-MD simulation results serving as the benchmark. Utilizing this CG force field, we conduct thermaldrawing MD simulations and thermal-conductivity calculations on bulk PE fibers to study the effects of thermal-drawing protocols, including the stretching (drawing) temperature, tensile strain level, and strain rate, on the observed enhancement in roomtemperature TCs. PE chain alignment and crystallinity (global and local) are quantified to discover the correlation between PE morphology (Fig. 1b) and TC.

More interestingly, we discover that a combination of moderate drawing temperature and strain rate is optimal in enhancing TCs by matching the temperature-dependent Rouse viscoelastic relaxation time and the applied strain rate. Such optimal thermal-drawing protocol is condensed to similar Deborah numbers (only differ across the melting temperature) over different drawing temperature and strain rate combinations considered here. Furthermore, phonon transport properties of thermally drawn PE fibers are computed to reveal the molecular mechanism behind the enhanced thermal transport. Using the effective-medium-theory model developed here based on serialparallel thermal resistors, we show that both chain alignment and crystallinity are important to TCs. Finally, we find that the greatly enhanced TC along the fiber-drawing direction is mainly attributed to the increased intrinsic phonon MFP, as well as the increased longitudinal phonon group velocities resulting from the enlarged elastic modulus along that direction.

\section{RESULTS AND DISCUSSION}

Chain alignment and degree of crystallinity of thermally drawn PE fibers

To reveal the impacts from fiber thermal-drawing conditions on polymer morphology and to develop a process-structure relationship, we investigated the chain alignment and crystallinity after thermal strains (see Methods). Figure 2 shows the orientational order parameters $P_{2 z}$ over all the beads after completing the 

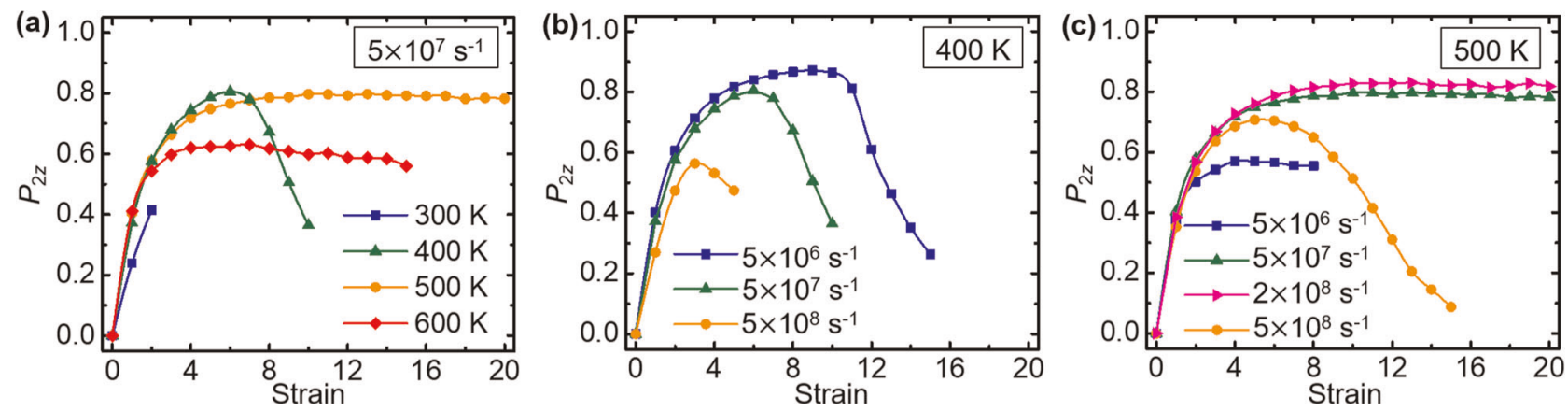

Fig. 2 The change of orientational order parameter of bulk PE as a function of strain obtained from different drawing temperatures and a strain rate of $5 \times 10^{7} \mathrm{~s}^{-1}$ a and a drawing temperature of $400 \mathrm{~K} \mathrm{~b}$ and $500 \mathrm{~K} \mathrm{c}$ and different strain rates followed by a 20 -ns relaxation at $300 \mathrm{~K}$.

thermal-drawing process outlined in Fig. 1c. At a drawing temperature of $500 \mathrm{~K}$ and a strain rate of $5 \times 10^{7} \mathrm{~s}^{-1}, P_{2 z}$ increases with increasing strain, reflecting more chain alignments (see the probability distribution of $P_{2 z}$ in Supplementary Fig. 1), and then reaches a plateau of $\sim 0.8$. However, under other conditions (e.g., a drawing temperature of $400 \mathrm{~K}$ and a strain rate of $\left.5 \times 10^{7} \mathrm{~s}^{-1}\right), P_{2 z}$ first increases and then decreases with increasing strain. This is different from the trend right after thermal drawing and before removing the external stresses, where $P_{2 z}$ increases with increasing the strain, and then reaches a plateau (Supplementary Fig. 2c), indicating partial reversibility of the thermal-drawing process upon removing the external stresses. The decrease in $P_{2 z}$ is probably attributed to excessive stresses built up under high strains (Supplementary Fig. 3b), the release of which results in structural shrinking and chain alignment destruction after external stresses (or strains) are removed. This also results from the increased volumes of nanovoid-type vacancies (Supplementary Fig. 4d), which provides room for chain folding after removing the strains from high strain levels.

In addition, $P_{2 z}$ also depends nonlinearly on the drawing temperature under the same strain rate. Figure 2a shows that $P_{2 z}$ reaches the highest values at intermediate drawing temperatures considered here (i.e., 400 and $500 \mathrm{~K}$ ). The tensile stress decreases with increasing the drawing temperature under the same strain level and strain rate (Supplementary Fig. 3a), reaching a steady value above $500 \mathrm{~K}$. On one hand, excessive stress concentration and vacancy formation during the tensile loading at lower drawing temperatures (e.g., $300 \mathrm{~K}$ ) are both harmful to chain alignment and would diminish $P_{2 z}$ (Supplementary Fig. 4b). On the other hand, higher tensile forces (stress) are still required to stretch polymer chains and compete against entropic chain randomization at higher temperatures. ${ }^{37}$ Therefore, lower stresses at higher drawing temperatures (i.e., $600 \mathrm{~K}$ ) fails to provide enough tensile forces to align the chains well, resulting in lower $P_{2 z}$.

Furthermore, strain rate also exhibits strong nonlinear influence on $P_{2 z}$ at the same drawing temperature. For example, under a drawing temperature of $400 \mathrm{~K}, P_{2 z}$ increases monotonically with decreasing the strain rate down to $5 \times 10^{6} \mathrm{~s}^{-1}$ (the smallest strain rate considered here due to the computational cost, see Fig. 2b). Under a lower drawing temperature of $300 \mathrm{~K}$, the PE fiber suffers from early fracture at small tensile strains of $\sim 2$, and therefore fails to align the polymer chains well (see the $P_{2 z}$ in Supplementary Fig. $5 a)$. Interestingly, under a drawing temperature of $500 \mathrm{~K}, P_{2 z}$ reaches a maximum under an intermediate strain rate of $2 \times 10^{8}$ $\mathrm{s}^{-1}$ (see Fig. 2c). A similar trend is observed under a higher drawing temperature of $600 \mathrm{~K}$ (Supplementary Fig. 5b), showing maximum $P_{2 z}$ under an intermediate strain rate of $5 \times 10^{8} \mathrm{~s}^{-1}$. It is noteworthy that the optimal strain rate to achieve the maximum $P_{2 z}$ increases from $5 \times 10^{6}$ to $5 \times 10^{8} \mathrm{~s}^{-1}$ when increasing the drawing temperature from 400 to $600 \mathrm{~K}$.
We further examined the impacts from fiber-drawing conditions on the resulting polymer crystallinity, which, interestingly, do not match exactly the trend observed in chain alignment $P_{2 z}$ especially under larger strains. Similar to $P_{2 z}$, the systemaveraged degree of crystallinity $\psi$ firstly increases with increasing the strain with local crystallites distributed uniformly in the bulk polymer (see Fig. 3a). Similar to $P_{2 z}, \psi$ reaches a maximum of $77 \%$ at an intermediate drawing temperature of $400 \mathrm{~K}$, as well as an intermediate strain rate of $5 \times 10^{7} \mathrm{~s}^{-1}$ (see Fig. 3b). Specifically, the degree of crystallinity increases with decreasing the strain rate down to $5 \times 10^{6} \mathrm{~s}^{-1}$ at a drawing temperature of $400 \mathrm{~K}$ (Fig. 3c). Similarly, the maximum degree of crystallinity also increases with decreasing the strain rate down to $5 \times 10^{6} \mathrm{~s}^{-1}$ under a drawing temperature of $300 \mathrm{~K}$ (Supplementary Fig. 6a). Note that it is challenging to further decrease the strain rate due to the high computational cost, and therefore we are not able to determine the optimal strain rate to maximize the degree of crystallinity under a drawing temperature of $300 \mathrm{~K}$. The degrees of crystallinity reach maxima under strain rates of $2 \times 10^{8}$ and $5 \times 10^{8} \mathrm{~s}^{-1}$, respectively, at drawing temperatures of 500 and $600 \mathrm{~K}$ (Fig. 3d, e).

The optimal strain rate to achieve the maximum crystallinity $\psi$ increases from $5 \times 10^{6}$ to $5 \times 10^{8} \mathrm{~s}^{-1}$ when increasing the drawing temperature from 400 to $600 \mathrm{~K}$, which agrees with the trend observed in $P_{2 z}$ (see Fig. 4). This is due to the fact that the alignment of polymer chains also enhances thread-like precursors responsible for the formation of the shish-kebab crystals, ${ }^{38}$ reflected from the further increase in $\psi$ after annealed stress relaxation, compared with the minor decrease in $\psi$ before annealed stress relaxation (Supplementary Fig. 7). Therefore, a combination of moderate drawing temperature and strain rate is essential to achieve both highest degrees of chain alignment and crystallinity. However, high polymer crystallinity does not guarantee perfect chain alignment (i.e., polymer chains in crystalline domains do not perfectly align) along the fiber-drawing direction, especially under high strain rates. This is reflected from the fact that $\psi$ does not drop off as much as $P_{2 z}$ under high strain rates. The structural shrinkage and chain alignment destruction after removing excessive stresses under high strains, which results in the drop off of $P_{2 z}$, however, does not affect $\psi$. Higher degrees of polymer crystallinity are expected to enhance their TC due to the higher TC in the crystalline phase (however, only along the chain direction) than that in the amorphous phase. ${ }^{25,39}$

Viscoelastic relaxation modulated chain alignment and crystallinity From the viscoelastic mechanics aspect, the tensile stress increases with increasing the strain rate under the same strain, as expected from the limited extent of stress relaxation during fast fiber drawing (Supplementary Fig. 3). In addition to the higher local stresses, higher strain rate will also lead to larger volume of vacancy in the form of nanovoids (Supplementary Fig. 4), which will further destroy the chain alignment. On the other hand, lower 
(a)

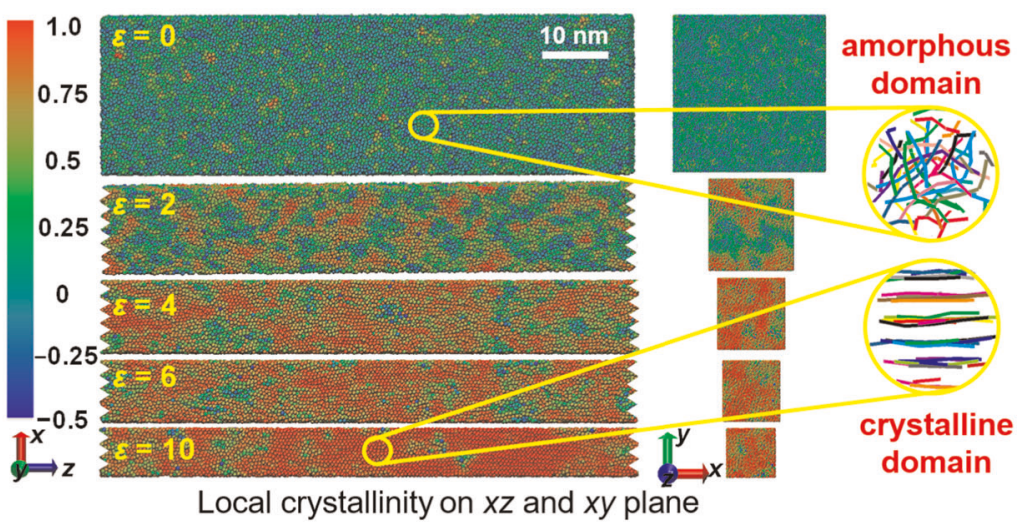

(b)

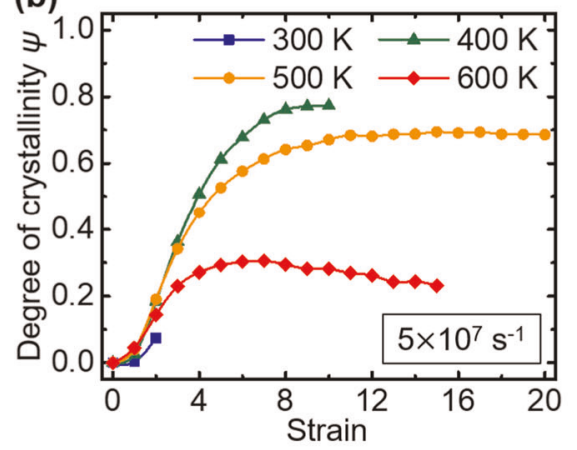

(c)

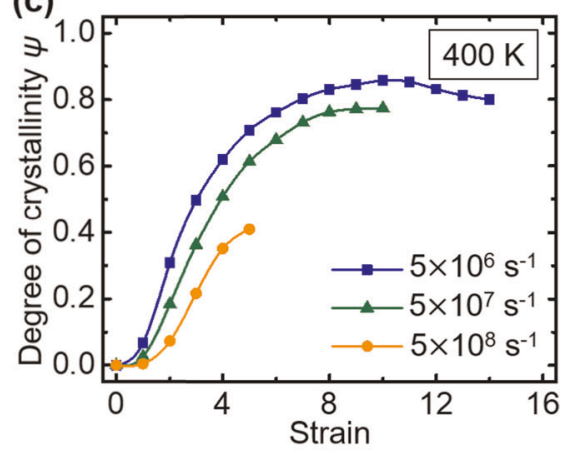

(e)
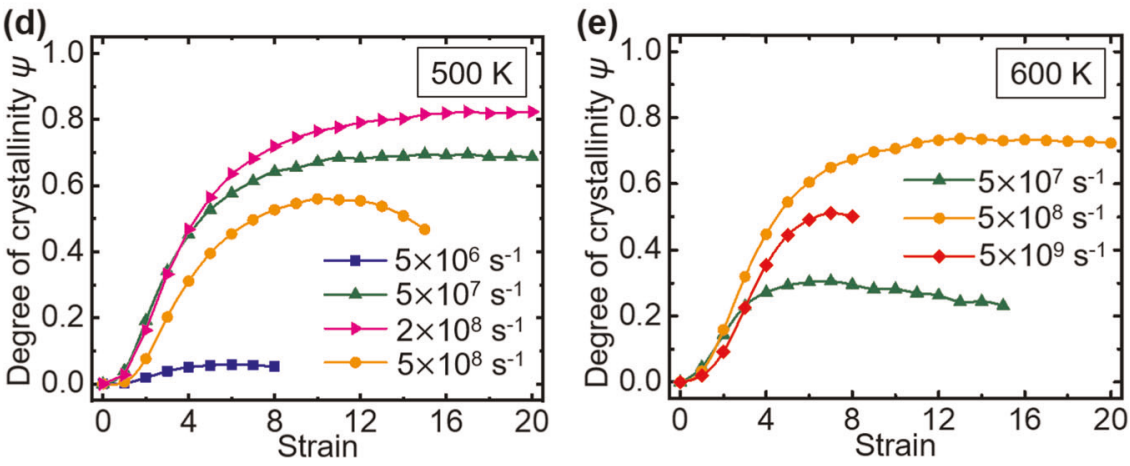

Fig. 3 Local crystallinity and system-averaged degrees of crystallinity $\boldsymbol{\psi}$ in thermally drawn PE fiber. a CG-MD simulation snapshots showing local crystallinity in thermally drawn PE fiber at strains $\varepsilon=0,2,4,6$, and 10 . Samples are obtained from a strain rate of $5 \times 10^{6} \mathrm{~s}^{-1}$ and a drawing temperature of $400 \mathrm{~K}$. The zoomed-in snapshots in a illustrate the morphology difference between the amorphous and crystalline domains. Each polymer bead is regarded as a crystalline bead when the local degree of crystallinity is $>0.7$. For strained samples, only the central regions of the simulation boxes are displayed. b System-averaged degrees of crystallinity $\psi$ with increasing the strain under a fixed strain rate of $5 \times 10^{7} \mathrm{~s}^{-1}$ and various drawing temperatures. c-e System-averaged degrees of crystallinity $\psi$ with increasing the strain under various strain rates and constant drawing temperatures of 400,500 , and $600 \mathrm{~K}$.

strain rate suffers from extra stress relaxation and unwanted configurational randomization of polymer chains, and therefore cannot provide enough tensile elongation forces to untie the entangled chains and align them further. In general, thermal energy transport along strong covalent bonds in well-aligned individual chains is more effective than that via weak van der Waals (vdW) interactions between different chains. Therefore, an optimal combination of fiber-drawing temperature and strain rate is essential to best align polymer chains and achieve the maximum TC along the fiber-drawing direction.

To unfold the viscoelastic mechanism behind the observed optimal drawing temperature and strain rate, we predicted the viscoelastic relaxation times for undrawn bulk PE at the above drawing temperatures (see Methods). The computed stress relaxation modulus $G(t)$ decays as a function of time and decays faster when increasing the temperature, as shown in Fig. 5. The plateau modulus of $G(t)$, defined after the linear Rouse dynamics ends and within the entanglement dynamics region in $G(t)$, is $\sim 2.5 \mathrm{MPa}$ at $400 \mathrm{~K}$ (see Supplementary Fig. 8) in this work, which matches the experimental value of $2.6 \mathrm{MPa}$ at $413 \mathrm{~K} .{ }^{40}$ The Rouse relaxation time $\tau_{\mathrm{R}}{ }^{41}$ which measures the diffusion time needed for chains shorter than the entanglement length to release stresses, is defined here when $G(t)$ decreases to $\sim 10^{7} \mathrm{~Pa}$. This cutoff in $G(t)$ is used to define $\tau_{R}$ because of the Rouse dynamics region, $G(t) \sim$ $t^{-0.5,37}$ and therefore the $\log (G(t))$ vs. $\log (t)$ curves remain linear with a constant slope for each temperature case (see Fig. 5), until $G(t)$ drops to $\sim 10 \mathrm{MPa}$ where the Rouse dynamics ends. Accordingly, $\tau_{\mathrm{R}}$ for PE fibers drawn at 400,500 , and $600 \mathrm{~K}$ are determined to be $5 \times 10^{-10}, 1 \times 10^{-10}$, and $5 \times 10^{-11} \mathrm{~s}$, respectively.

To verify the computed Rouse relaxation time $\tau_{R}$, we have converted the dimensionless $\tau_{\mathrm{R}}$ of polymers, predicted by 

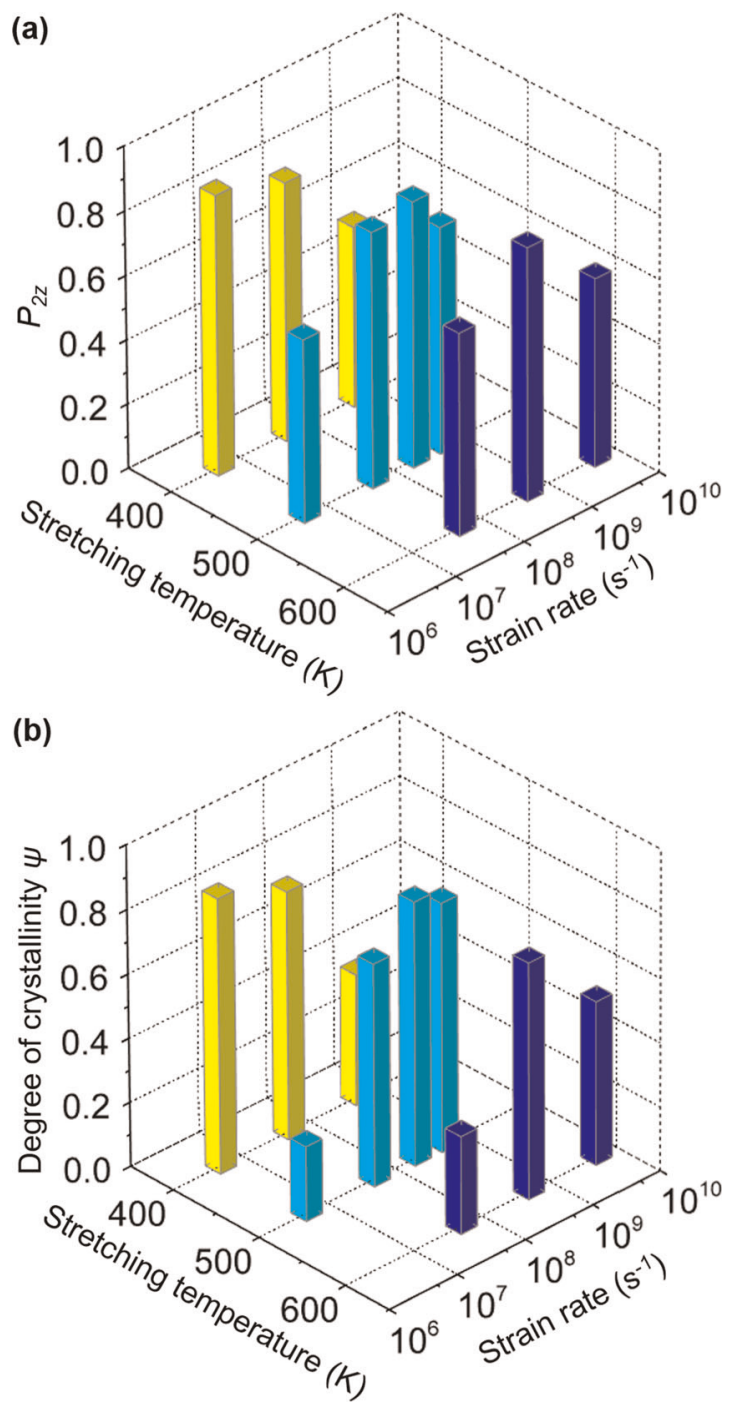

Fig. 4 The maximum strain-induced $P_{2 z}$ a and degree of crystallinity $\psi \mathbf{b}$ achieved after annealed stress relaxation as a function of fiberdrawing temperature and strain rate. The optimal strain rates to achieve maximum $P_{2 z}$ and degree of crystallinity at 400,500, and $600 \mathrm{~K}$ are $5 \times 10^{6}, 2 \times 10^{8}$, and $5 \times 10^{8} \mathrm{~s}^{-1}$, respectively.

Likhtman et al. ${ }^{41}$ using MD simulations and dimensionless force field units (in which the dimensionless time is normalized by $t^{*}=\sigma \sqrt{\frac{m}{k_{B} T}}$, to the physical $\tau_{\mathrm{R}}$ values presented using real force field units here. A dimensionless $\tau_{R}$ of 1000 for the 100-bead polymer melt at $365 \mathrm{~K}$ by Likhtman et al. ${ }^{41}$ is converted into a physical $\tau_{\mathrm{R}}$ of $2.4 \times 10^{-9} \mathrm{~s}$ based on the $\sigma, m$, and $T$ values used here. This is within an order of magnitude from our computed $\tau_{R}$ values of $5 \times 10^{-10} \mathrm{~s}$ at $400 \mathrm{~K}$ for the 150 -bead PE melt in this work. The differences could be due to the differences in the temperatures applied, the molecular weights of the polymers simulated, and the functional forms used in the two force fields. Therefore, our $\tau_{\mathrm{R}}$ predictions could be considered reasonably accurate when compared to other theoretical studies.

The decrease in $\tau_{R}$ correlates perfectly well with the increase in the optimal strain rates when increasing the drawing temperature from 400 to $600 \mathrm{~K}$. This is due to the fact that when the stress relaxes faster, shorter processing time (i.e., higher strain rate) is required to lock stresses for aligning polymer chains under higher temperatures. Excessively low strain rates lead to a liquid-like polymer, which diffuses too fast into disordered structures, while

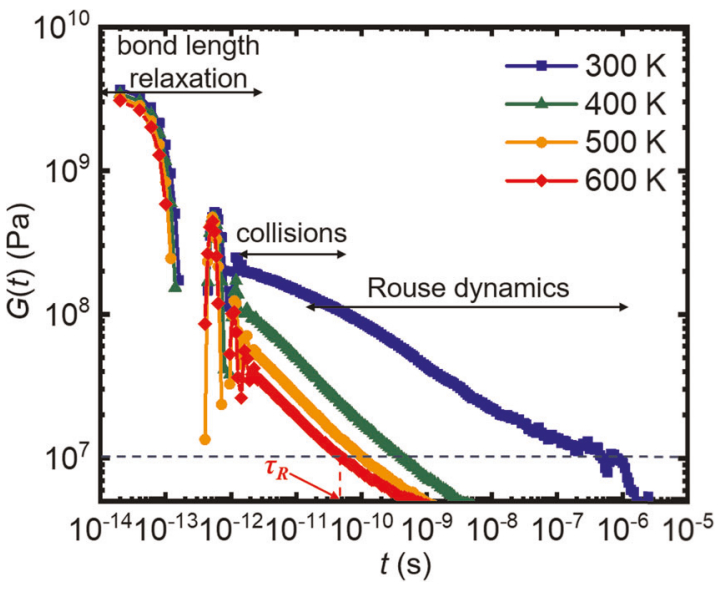

Fig. 5 Time evolutions of stress relaxation moduli, $G(t)$, for undrawn amorphous $\mathrm{PE}$ at various temperatures. The various stages of chain relaxation are denoted in the figure.

excessively high strain rates lead to a solid-like polymer, which is easy to break and form nanovoids. These two competing factors indicate that the optimal combination of drawing temperature and strain rate, a delicate balance between stress localization and chain diffusion (for viscoelastic relaxation), is critical for the polymer thermal-drawing process.

With the above in mind, we utilize the Deborah number $D_{e}{ }^{42}$ a dimensionless number measuring the competing effects between chain diffusion (which leads to viscoelastic relaxation) and stress localization (which helps chain alignments) rates, to condense the optimal drawing temperature (which is reflected by the corresponding viscoelastic relaxation time) and strain rate into a single value. $D_{\mathrm{e}}$ is defined here as the ratio between the temperaturedependent Rouse relaxation time $\tau_{R}$ and the thermal-drawing processing time $\tau_{\mathrm{p}}$. Note that $\tau_{\mathrm{p}}$, estimated by the simulation time when TC reaches the maximum under tensile loading, is a good measure of the stress localization rate and inversely correlated with the strain rate. To achieve optimal strain rates at drawing temperatures of 400,500 , and $600 \mathrm{~K}, \tau_{\mathrm{P}}$ (see Fig. $7 \mathrm{~b}-\mathrm{d}$ ) are $2 \times$ $10^{-6}, 1 \times 10^{-7}$, and $3.8 \times 10^{-8} \mathrm{~s}$, respectively. The corresponding values of $D_{\mathrm{e}}$ are $2.5 \times 10^{-4}, 1 \times 10^{-3}$ and $1.3 \times 10^{-3}$, respectively. Although we have not exhaustively tested other drawing temperature and strain rate combinations here, we could imply from the current data that a $D_{\mathrm{e}}$ in the order of $1 \times 10^{-3}$ would lead to the maximum degrees of chain alignment and crystallinity for thermal-drawing above the melting temperature ( $>409 \mathrm{~K}$ for PE here, see Supplementary Discussion and Supplementary Fig. 19). For the thermal-drawing process below the melting temperature, a much smaller $D_{\mathrm{e}}$ in the order of $1 \times 10^{-4}$ would be optimal to process a glassy polymer fiber due to significantly slower chain diffusions (measured by $\tau_{\mathrm{R}}$ ).

To validate the magnitude of the $D_{\mathrm{e}}$ measured in this work, we estimated and compared to $D_{\mathrm{e}}$ in Shrestha et al.'s experimental work. ${ }^{13}$ In that work, the PE microfiber was locally drawn under a strain rate of $1400 \mathrm{~s}^{-1}$ and a drawing temperature of $450 \mathrm{~K}$. The strain (or draw-down ratio) of PE was estimated to be 225 because the diameter of the PE fiber deceases from 1.5 to $100 \mathrm{~nm}$. Therefore, the processing time should be $225 / 1400=0.16 \mathrm{~s}$. The molecular weight $M$ of PE in Shrestha et al.'s work is $3-6 \times 10^{6} \mathrm{~g}$ $\mathrm{mol}^{-1}$. The Rouse relaxation time $\tau_{\mathrm{R}}$ follows a power scaling law of $\tau_{\mathrm{R}} \sim M^{2}{ }^{37}$ Considering that our computed $\tau_{\mathrm{R}}$ values are reasonably accurate, we then used the above-scaling law to estimate the $\tau_{R}$ value for PE with a molecular weight of $M=4.5 \times 10^{6} \mathrm{~g} \mathrm{~mol}^{-1}$ studied in Shrestha et al.'s work. The $\tau_{\mathrm{R}}$ in Shrestha et al.'s work is estimated to be $\sim 6 \times 10^{-5} \mathrm{~s}$, based on $\tau_{\mathrm{R}}$ values of $5 \times 10^{-10}$ and $1 \times 10^{-10} \mathrm{~s}$ at 400 and $500 \mathrm{~K}$, respectively, with $M=10,500 \mathrm{~g}$ 

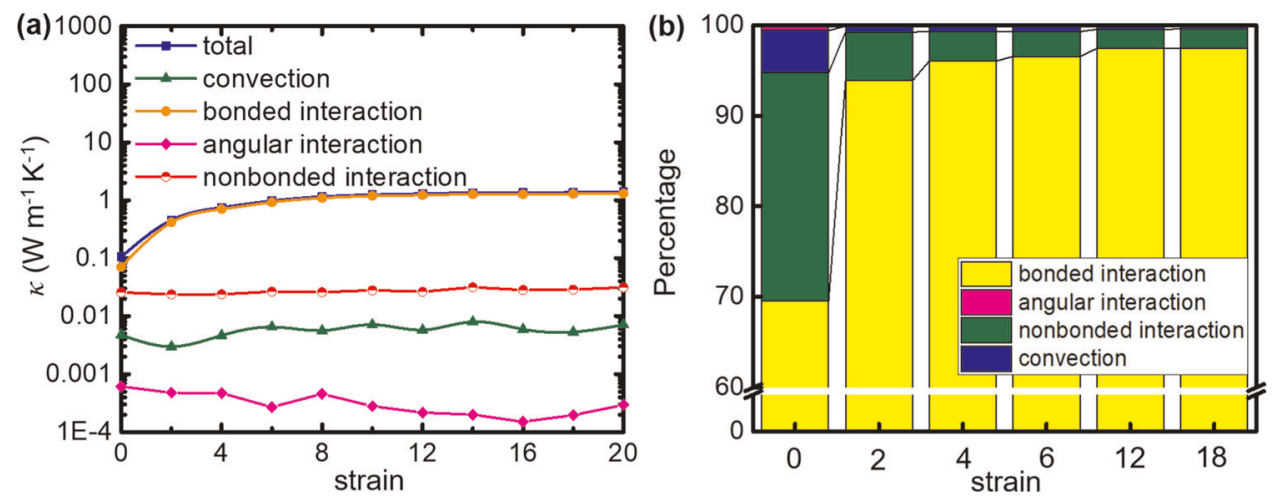

Fig. 6 TC contributions from the virial (bonded, angular, and nonbonded interactions) and the convection terms a and their ratios with respect to the total TC $\mathbf{b}$ as a function of strain under a drawing temperature of $500 \mathrm{~K}$ and a strain rate of $5 \times 10^{7} \mathrm{~s}^{-1}$.

$\mathrm{mol}^{-1}$ used here. As a result, $D_{\mathrm{e}}$ in Shrestha et al.'s work is estimated to be $6 \times 10^{-5} \mathrm{~s} / 0.16 \mathrm{~s}=3.75 \times 10^{-4}$, which matches reasonably well with our computed $D_{\mathrm{e}}$ of $2.5 \times 10^{-4}$ and $1 \times 10^{-3}$ at 400 and $500 \mathrm{~K}$, respectively, to achieve the optimal fiber morphology and TC. These interesting findings suggest that it would take around $10^{3}$ and $10^{4}$ times of the Rouse relaxation time of polymers of different molecular weights, respectively, to optimally draw a polymer fiber in the melt and the glassy state.

\section{Impacts from fiber-drawing conditions on TC}

In general, the TC of bulk PE along the drawing direction increases with increasing the strain (a few exceptions are discussed below), as shown in Fig. 6a. Contributions to the total TC include those from the virial (i.e., bonded, angular, and nonbonded interactions here) and the convection (kinetic energy) terms (see Methods). Figure 6 a shows that the contribution from bonded interactions to the total TC is dominant and also increases with increasing the strain, which is expected, as high-frequency (energy) phonons are carried by bond vibrations. The contribution from nonbonded interactions to the total TC ranks second, but is almost independent of strain. Contributions from angular interactions and convections to the total TC are both negligible. As the strain increases, the contribution from bonded interactions to the total TC increases from 69 to $97 \%$, while that from nonbonded interactions decreases from 25 to only $2 \%$ (Fig. 6b). This finding is reasonable because the backbones of polymer chains reorient towards the drawing direction with increasing the strain, such that heat can transfer in parallel to the backbones more efficiently through stronger bonded interactions rather than weaker vdW interactions.

In addition, we observe that the TC of bulk PE along the stretching direction correlates well with both the chain alignment $P_{2 z}$ and the degree of crystallinity $\psi$, since both morphological features are enhanced with increasing the strain, except for the cases under high strain rates. For example, as shown in Fig. 7a, under an intermediate strain rate of $5 \times 10^{7} \mathrm{~s}^{-1}$, TC reaches maximum at an intermediate drawing temperature of $500 \mathrm{~K}$. A nonlinear trend between TC and the strain level is observed under high strain rates, when both $P_{2 z}$ and $\psi$ drop off due to limited stress relaxation and the resulting internal structural defects. For example, TC decreases with further elongation at a drawing temperature of $400 \mathrm{~K}$ and all the strain rates considered here, as shown in Fig. 7b.

Similar to the trend observed in chain alignment $P_{2 z}$ and degree of crystallinity $\psi$, moderate strain rate and drawing temperature are both essential to the improvement of TC, as shown in Fig. 7. Lower optimal strain rates for TC are achieved under lower drawing temperatures, a trend that can be explained by the delicate balance between viscoelastic relaxation and stress localization, where such optimal combination can be condensed into similar Deborah numbers (see above). Among all the drawing conditions considered here, TC reaches a maximum of $1.57 \mathrm{~W}$ $\mathrm{m}^{-1} \mathrm{~K}^{-1}$ at a strain level of 20 , a drawing temperature of $500 \mathrm{~K}$, and a strain rate of $2 \times 10^{8} \mathrm{~s}^{-1}$, which is 14 times higher than that of undrawn amorphous PE. Such TC achieved through thermal drawing is outstanding, reaching $80 \%$ of the theoretical limit of bulk $\mathrm{PE}$, that is, its pure crystalline counterpart with $\mathrm{TC} \kappa_{\mathrm{c} \|}=$ $1.953 \mathrm{~W} \mathrm{~m}^{-1} \mathrm{~K}^{-1}$ parallel to the chains in a PE crystal (see below for details). It is important to note that achieving the theoretical TC limit of a single PE chain in bulk PE could be challenging due to excessive inter-chain phonon scatterings due to vdW-type weak nonbonded interactions.

However, neither $P_{2 z}$ nor $\psi$ can correlate perfectly well with TC to yield a predictive model using only one of these two structure ordering parameters. For example, TC data scatter around with respect to $P_{2 z}$, although they do show some exponential increase with increasing $P_{2 z}$, which agree with the model proposed by Liu and Yang, ${ }^{22}$ but with many high TC outliers (Fig. 8a). Similarly, TC data also scatter around with respect to $\psi$, showing weak increasing trend with increasing $\psi$ (Fig. 8b). Since both factors have significant impacts on the reported TC here along PE fibers, we need to develop a new model to correlate TC with both $P_{2 z}$ and $\psi$.

Based on the effective-medium theory, ${ }^{43}$ we developed a new model here for the TC of semicrystalline polymers under tensile deformation. Note that the Choy-Young's model ${ }^{39}$ and the series model $^{44,45}$ underestimate the TC at high degrees of crystallinity. ${ }^{46}$ The reason is that both models consider the TC of the amorphous domain as a constant. However, the TC of amorphous domain should change with strain due to the corresponding change in the orientational order parameters of PE chains in the amorphous domain (see Supplementary Fig. 9). In addition, the orientational distribution of PE chains along the drawing direction is not uniform due to the local residue stresses, and therefore the thermal resistors in a model should vary along the drawing direction. With the above in mind, an accurate effective-medium theory should include the above two factors. On the other hand, measuring the bridge numbers and the TC of each bridge are challenging in the modified Takayanagi's model with varying thermal resistors, ${ }^{46}$ so the PE morphology information obtained from CG-MD simulations here would be very useful in developing such a model.

In our effective-medium theory model, the simulated polymer system is divided into $N_{\mathrm{s}}$ serial slabs along the drawing direction to address the issue of non-uniform chain orientations, with the thickness of each slab equal to the diameter of a CG bead $(5.8 \AA$ ). Each slab is composed of both crystalline and amorphous domains, mimicking thermal resistors in parallel, due to the fact that they blend well with each other, as shown from the crosssectional area of the PE fibers (Fig. 3a). This new model combines 

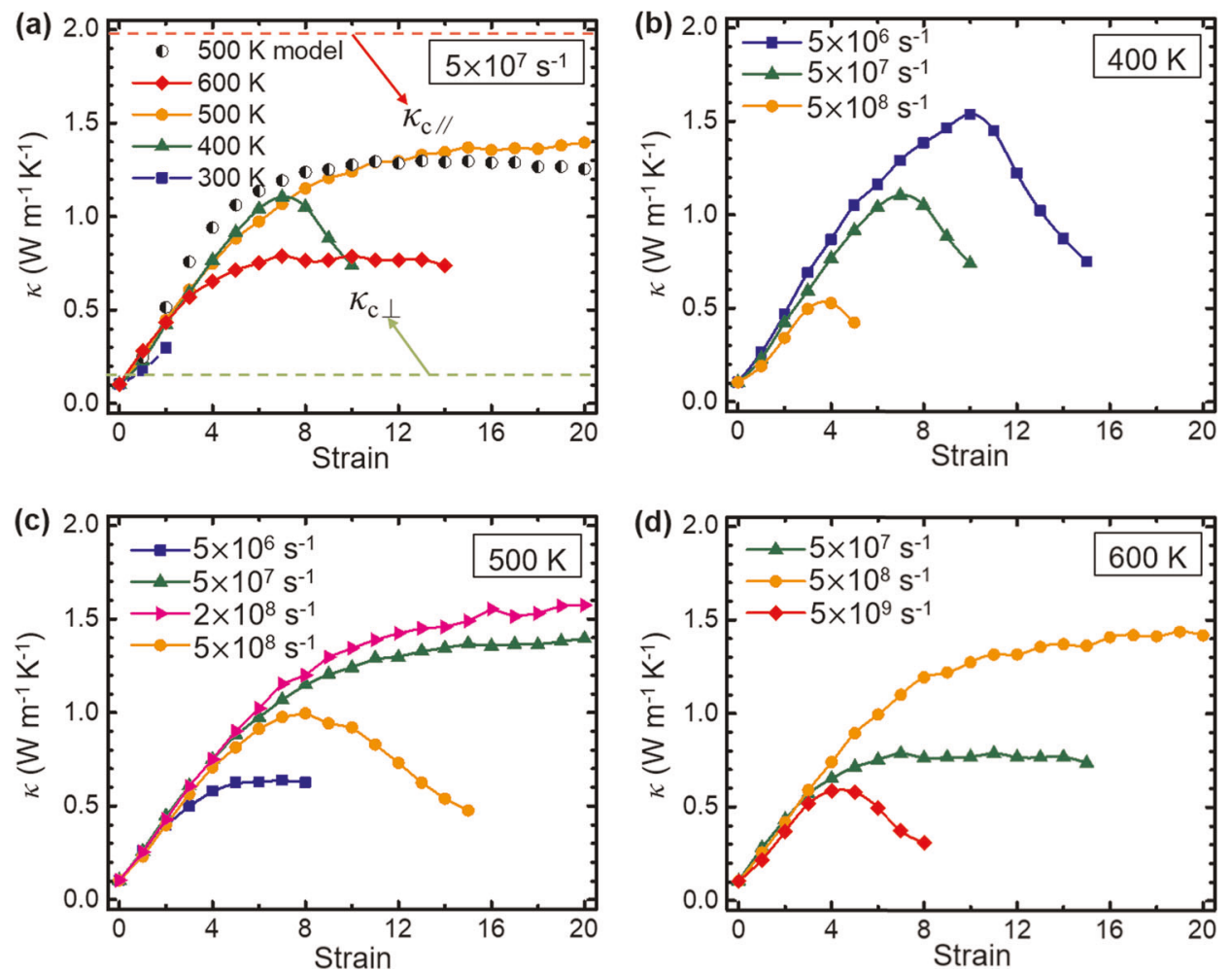

Fig. 7 TC of bulk PE along the drawing direction. a TCs of bulk PE as a function of strain under various drawing temperatures and a fixed strain rate of $5 \times 10^{7} \mathrm{~s}^{-1}$. TCs as a function of strain under various strain rates and fixed drawing temperatures of b $400 \mathrm{~K}$, c $500 \mathrm{~K}$, and $\mathbf{d} 600 \mathrm{~K}$. The half black and white circles in a denote the model-predicted TC values based on the effective-medium theory developed here.

serial and parallel thermal resistors systematically, as illustrated in Fig. 9a. Finally, the effective TC $\kappa_{\text {eff }}$ along the drawing direction is estimated by the following equation:

$\frac{1}{K_{\mathrm{eff}}}=\sum_{i=1}^{N_{\mathrm{s}}}\left[\Psi_{i}\left(\kappa_{\mathrm{c} \perp} \frac{2-2 P_{2 z \mathrm{c}}^{i}}{3}+\kappa_{\mathrm{c} \|} \frac{2 P_{2 z \mathrm{c}}^{i}+1}{3}\right)+\left(1-\Psi_{i}\right) \kappa_{0} \exp \left(a P_{2 z \mathrm{a}}^{i}\right)\right]^{-1}$,

where $\psi_{i}$ is the degree of crystallinity of the ith slab; $\kappa_{\mathrm{c} \|}$ and $\kappa_{\mathrm{c} \perp}$ are the TCs of the crystalline domain in parallel and perpendicular to the fiber axis, respectively; $P_{2 z c}^{i}$ and $P_{2 z a}^{i}$ are the orientational order parameters over crystalline and amorphous beads in the ith slab, respectively; $\kappa_{0}$ is the TC of undrawn isotropic amorphous PE; and $a$ is the only fitting parameter obtained by minimizing the relative deviations between CG-MD simulated and modelpredicted TCs under all the conditions here. Note that the exponential correlation between the TC contribution from the amorphous domain and $P_{2 z a}^{i}$ on the right of Eq. 1 is based on the model developed by Liu and Yang, 22 where pre-factor $a$ was introduced as a fitting parameter.

To determine $\kappa_{\mathrm{c} \|}$ and $\kappa_{\mathrm{c} \perp}$, we conducted equilibrium molecular dynamics (EMD) simulations (see Methods) using the Green-Kubo method. ${ }^{47}$ A perfect PE crystal structure consisting of 36 150-bead polymer chains is used here and shown in Supplementary Fig. 10a. To obtain reliable predictions of $\kappa_{\mathrm{c} \|}$ and $\kappa_{\mathrm{c} \perp}$, EMD simulations were repeated five times using different random seeds for generating the initial bead velocities. The size dependence of $\kappa_{c \|}$ is also tested by studying a simulation cell with double the length along the chain axis, and no discernible size dependence is found. In addition, due to the low energy carried by long wavelength phonons in polymers, we do not expect the long wavelengthcutout effect in our EMD simulations with a large scale of $\sim 100 \mathrm{~nm}$ to greatly affect the TC results. The resulting ensemble-averaged $\kappa_{\mathrm{c} \|}=1.953 \mathrm{~W} \mathrm{~m}^{-1} \mathrm{~K}^{-1}, \kappa_{\mathrm{c \perp}}=0.131 \mathrm{~W} \mathrm{~m}^{-1} \mathrm{~K}^{-1}, \kappa_{0}=0.105 \mathrm{~W} \mathrm{~m}^{-1}$ $\mathrm{K}^{-1}$, and the fitting parameter $a=1.5$. Note that $\kappa_{0}$ of $0.105 \mathrm{~W}$ $\mathrm{m}^{-1} \mathrm{~K}^{-1}$ for unstrained amorphous PE is lower than those of semicrystalline PE measured in experiments due to both the absence of crystallinity and the relatively low molecular weight considered here. Our predicted $\kappa_{0}$ value does match the experimental value of $\sim 0.11 \mathrm{~W} \mathrm{~m}^{-1} \mathrm{~K}^{-1}$ for fully amorphous polymers well. ${ }^{48}$

The model-predicted TC profile under a drawing temperature of $500 \mathrm{~K}$, strain rate of $5 \times 10^{7} \mathrm{~s}^{-1}$, and different strain levels (see Fig. 7a) agrees well with the CG-MD simulated TC profile. For all thermal-drawing conditions and strain levels, relative deviations between the simulated and the model-predicted TCs (see Fig. 9b) are mostly within $20 \%$, except for a few cases under excessively high strain rates and strain levels. For example, under a drawing temperature of $600 \mathrm{~K}$, strain rate of $5 \times 10^{9}$, and strain levels of 7 and 8 , the relative deviations are $\sim 50 \%$. Excluding these outliers, the average relative deviation is $\sim 11.5 \%$ over all the thermaldrawing conditions.

\section{Enhanced phonon transport properties from crystallization}

To elucidate the underlying mechanism behind thermal transport in thermally drawn polymer fibers, we investigate the phonon properties of PE samples at $300 \mathrm{~K}$. Without loss generality, here we consider samples under different strain levels prepared at a representative strain rate of $5 \times 10^{7} \mathrm{~s}^{-1}$ and a drawing temperature of $500 \mathrm{~K}$. According to the kinetic theory of phonon transport, the TC can be estimated as: ${ }^{47}$

$\kappa=\frac{1}{V} \sum_{p, k} C_{p, k} v_{p, k} I_{p, k}=\frac{1}{3 V N_{0}} \sum_{p, k} \hbar \omega_{p, k} D_{p, k} \frac{\partial f_{p, k}}{\partial T} v_{p, k} I_{p, k}$,

where $C_{p, k}$ is the phonon specific heat, $v_{p, k}$ is the group velocity, $I_{p, k}$ is the phonon MFP, $V$ is the volume of the sample, $N_{0}$ is the total number of beads in the system, $D_{p, k}$ is the phonon vibrational density of states (VDOS), and $f_{p, k}$ is the Bose-Einstein distribution function for phonon excitations. The subscripts " $p$ " and " $k$ " indicate 

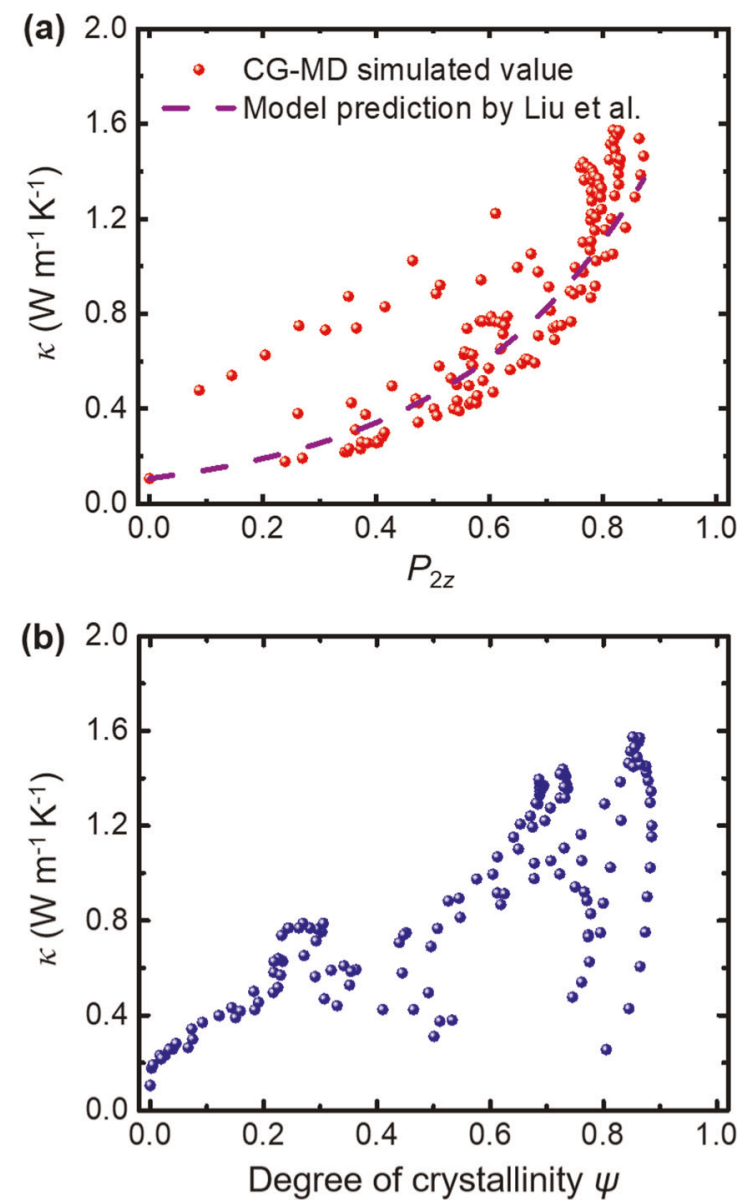

Fig. 8 Variations of calculated TC as a function of a the orientational order parameter $P_{2 z}$ and $\mathbf{b}$ the degree of crystallinity $\psi$. The modelpredicted TC profile by Liu and Yang ${ }^{22}$ is shown as a dashed line in a for comparison.

phonon polarization and wave vector, respectively. Equation 2 unveils that the mode-specific phonon MFP, VDOS, and group velocity are the primary factors dictating phonon thermal transport. Note that $I_{p, k}$ could be decomposed into contributions from the intrinsic phonon-phonon scattering $I_{\mathrm{ph}-\mathrm{ph}}$ and the phonon-boundary scattering $I_{\mathrm{ph}-\mathrm{b}}$ due to the finite simulation box dimension.

Figure 10a shows the calculated intrinsic phonon MFP $I_{\text {ph-ph }}$ and the intrinsic TC for a macroscopic sample $\kappa_{\infty}$, obtained from length-dependent TC results where the impact from the finite system size-induced phonon-boundary scattering is removed from NEMD simulations (see Methods and Supplementary Fig. 11). Through tensile deformation, $I_{\mathrm{ph}-\mathrm{ph}}$ increases from around $10 \AA$ at zero strain to $3367 \AA$ at a strain of 18 . This is due to the fact that strain-induced chain alignment and crystallization reduce structural disorders, weaken three-phonon Umklapp scattering, and thus, enhance $I_{\text {ph-ph. }}$.

The top of Fig. 10b shows the phonon VDOS of amorphous PE under different strain levels and that of a perfect PE crystal consisting of 2025 150-bead chains (structure shown in Supplementary Fig. 10b). When increasing the strain, the two major peaks, originally located at 5 and $27 \mathrm{THz}$, in the VDOS shift slightly to the right. In addition, when increasing the strain, the height of the first peak increases, while that of the second peak decreases. The VDOS peak at $27 \mathrm{THz}$ is attributed to the vibrations of covalent bond, $\omega_{\text {bond, }}$ based on the simple normal mode equation: $\omega_{\text {bond }}=2 \sqrt{k_{e} / m}$, where $k_{e}$ is the force constant of the bond stretching potential and $m$ is the mass of the bead. The VDOS

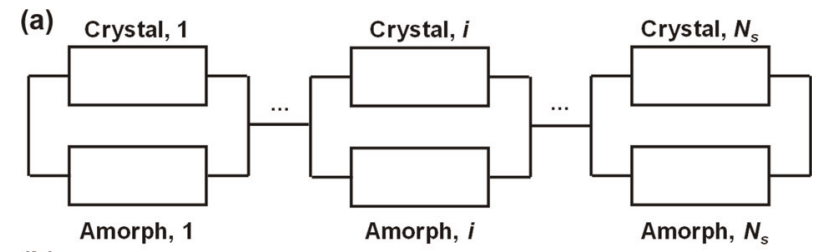

(b)

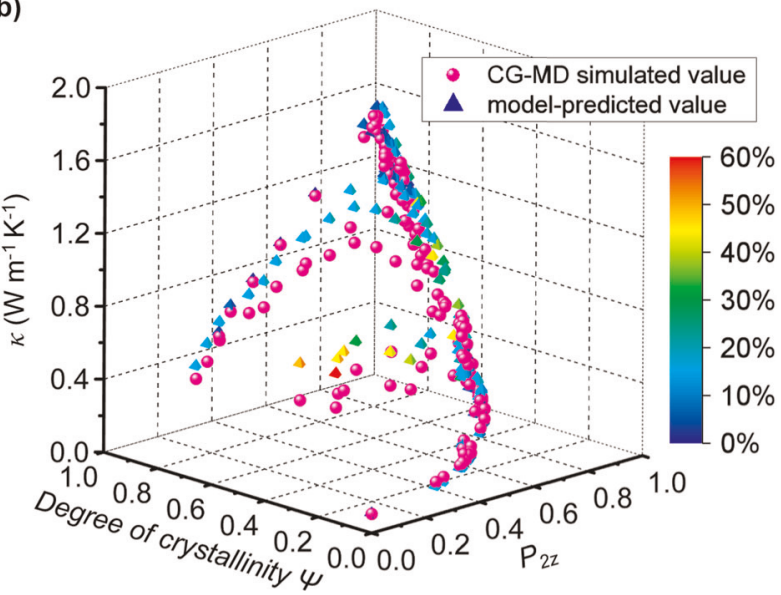

Fig. 9 Theoretical model of TC and comparison between simulated and model-predicted TCs. a Illustration of the effective-medium theory developed here based on a serial-parallel thermal resistor model for the TC of semicrystalline polymers under tensile deformations. The $N_{s}$ slabs, each composed of crystalline and amorphous domains in parallel, are serially connected to each other. b 3D plots of CG-MD simulated and model-predicted TCs under different $\psi$ and $P_{2 z}$. The color of the triangular symbols represents the relative deviations (in absolute values) between CG-MD simulated and model-predicted TCs.

peak at $5 \mathrm{THz}$ thus comes from angular bending and nonbonded interactions. The above trends in VDOS are expected, as the VDOS of amorphous PE under larger strain converges to that of a perfect PE crystal. According to Eq. 2, the frequency-dependent specific heat approximately correlates with the product of frequency and VDOS. The bottom of Fig. 10b shows that phonon specific heat in low-frequency domain increases, while that in high-frequency domain decreases when increasing the strain. These differences in frequency-dependent VDOS and specific heat are expected to have minor impacts on TCs of thermally drawn PE fibers.

Figure $10 \mathrm{c}$ shows that the longitudinal group velocity $v_{\mathrm{L}}$ increases when increasing the strain, while the transverse group velocity $v_{\mathrm{T}}$ is not greatly affected by the strain (see Methods). Through tensile deformation, $v_{L}$ at a strain of 18 is three times larger than that at zero strain due to the enhanced elastic modulus along the fiber-drawing direction. Therefore, the increase in TC is mainly attributed to the improvements in the intrinsic phonon MFP and the longitudinal group velocity, while the phonon VDOS has a minor contribution to the enhancement in TC.

In summary, a predictive CG-MD model for simulating the thermal-drawing process and the TC of PE fibers was developed here. This carefully trained model, facilitated by target property matching and the MS-IBI method, could successfully describe the structural, phase transition, and TC properties of PE fibers. CG-MD simulations are performed to investigate the impacts of annealed tensile deformation (mimicking fiber-drawing conditions, such as drawing temperatures, strain levels, and strain rates) on chain alignment, crystallinity, phonon transport, and the resulting TCs in bulk PE. Our simulation results show that $P_{2 z}$, a quantitative measure of chain alignment, increases and then reaches a plateau with increasing the strain, except for the cases under excessively 
(a)

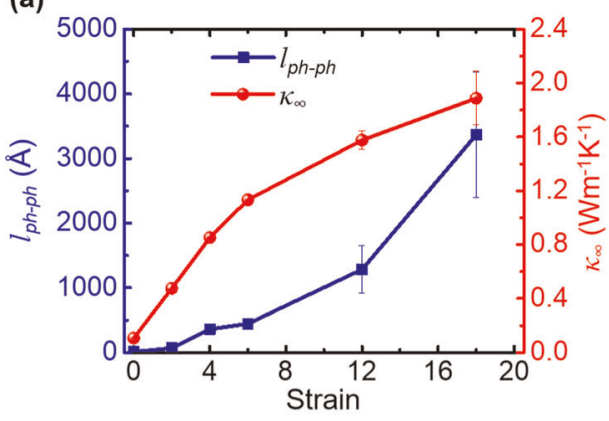

(b)

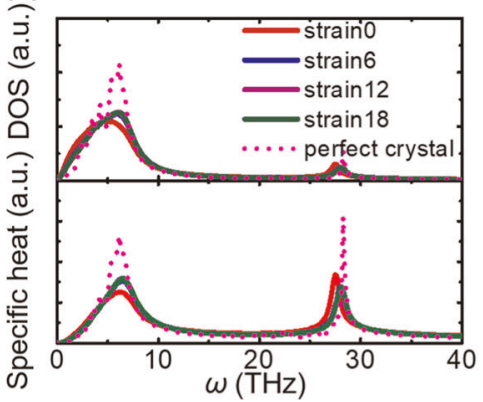

(c)

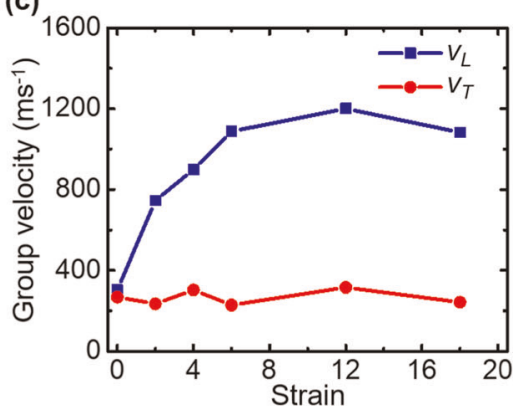

Fig. 10 Intrinsic phonon transport properties of bulk PE at $300 \mathrm{~K}$ with increasing strain under a strain rate of $5 \times 10^{7} \mathrm{~s}^{-1}$ and a drawing temperature of $500 \mathrm{~K}$. a Phonon intrinsic mean free path $I_{\mathrm{ph}-\mathrm{ph}}$ and intrinsic TC $\kappa_{\infty}$. The error bars represent standard errors from linearly fitting the $1 / K(L)$ vs. $1 / I_{\text {ph-b }}$ curve (see Supplementary Fig. 11). b Phonon VDOS (top) and the corresponding spectral specific heat (bottom). c Longitudinal $\left(v_{\mathrm{L}}\right)$ and transverse $\left(v_{\mathrm{T}}\right)$ group velocities.

Table 1. Thermal-drawing processing parameters for bulk PE fibers considered here.

\begin{tabular}{ll}
\hline Drawing temperatures $(\mathrm{K})$ & Strain rates $\left(\mathrm{s}^{-1}\right)$ \\
\hline 300 & $5 \times 10^{6}, 5 \times 10^{7}$ \\
400 & $5 \times 10^{6}, 5 \times 10^{7}, 5 \times 10^{8}$ \\
500 & $5 \times 10^{6}, 5 \times 10^{7}, 2 \times 10^{8}, 5 \times 10^{8}$ \\
600 & $5 \times 10^{7}, 5 \times 10^{8}, 5 \times 10^{9}$ \\
\hline
\end{tabular}

high strain rates, where $P_{2 z}$ increases and then drops off sharply due to extreme stress concentrations and the resulting structural damage. Likewise, the degree of crystallinity, $\psi$, shows similar tendency to $P_{2 z}$ with respect to strain, but differs from $P_{2 z}$ under high strain levels (i.e., $\psi$ only drops slightly). Both $P_{2 z}$ and $\psi$ exhibit nonlinear dependences on the drawing temperature and strain rate. Lower drawing temperatures will lead to slower stress relaxation, and possibly destroy the chain alignment and crystallinity after releasing the residual stress, while excessively high drawing temperatures cannot provide enough tensile forces (stress dissipates too fast due to chain diffusions) to align polymer chains well or promote crystallization. Meanwhile, higher strain rates will lead to extreme stress localization and structural damage, similar to the cases at lower temperatures, while lower strain rates suffer from extra stress relaxation and chain diffusions, similar to the cases at higher temperatures.

An optimal combination of moderate drawing temperature and strain rate is essential to achieve highest degrees of chain alignment $P_{2 z}$, crystallinity $\psi$, and the resulting TCs. From the viscoelastic relaxation aspect, we found that such an optimal combination requires a delicate balance between stress localization (to align polymer chains) and chain diffusion (for viscoelastic relaxation). After examining the Deborah number, we found that it would take around $10^{3}$ and $10^{4}$ times of the Rouse relaxation time of melt and glassy polymers (with the molecular weight considered here), respectively, to achieve the optimal fiber performance. The Deborah number could serve as an important and general dimensionless metric to design the best thermaldrawing protocols for a wide range of polymer types under various combinations of drawing temperature and strain rate.

For phonon transport, the contribution from bonded interactions to the total TC is more significant than contributions from nonbonded interactions, angular interactions and convections. The influences of strain level, drawing temperature, and strain rate on TC are similar to those on $P_{2 z}$ and $\psi$. However, neither $P_{2 z}$ nor $\psi$ can correlate very well with the computed TC due to the interplay between $P_{2 z}$ and $\psi$. An effective-medium-theory model, considering impacts from both $P_{2 z}$ and $\psi$, as well as the serial-parallel nature of semicrystalline polymers, has been successfully applied here to predict the TCs of thermally drawn PE fibers. Furthermore, the enhancement in TC by tensile deformation is mainly attributed to the increases in the intrinsic phonon MFP and the longitudinal group velocity. We believe that this large-scale molecular modeling work could provide fundamental insights into the thermal-drawing process of semicrystalline polymer fibers and provide a complete process-structure-property correlation. This work could also guide future experimental efforts to optimize thermal-drawing protocols to promote chain alignment, degree of crystallinity, and the resulting phonon transport performance.

\section{METHODS}

Modeling the annealed fiber-drawing process via thermal strains All the MD simulations are conducted using the LAMMPS software package ${ }^{49}$ with periodic boundary conditions in three dimensions. The CG force field for $\mathrm{PE}$ is developed based on AA-MD simulation results using the AIREBO potential. ${ }^{50}$ For the CG force field training approach and validation results, please refer to Supplementary Method and Supplementary Discussion. To investigate the polymer fiber-drawing process modeled via thermal straining, a system consisting of 2025 150-bead PE chains was relaxed at $500 \mathrm{~K}$ using the double-bridging ${ }^{51}$ algorithm for $200 \mathrm{~ns}$. The randomized amorphous PE system was first quenched to $300 \mathrm{~K}$ at a rate of $2 \mathrm{~K} \mathrm{n} \mathrm{s}^{-1}$, and then heated to $600 \mathrm{~K}$ at the same rate, during which samples at different temperatures were collected. After preparing amorphous PE samples under 300, 400, 500, and 600 K, CG-MD tensile deformation simulations were performed at the above temperatures under various strain rates (see Table 1). Note that the strain rates here are higher than experimental values, for example, $1400 \mathrm{~s}^{-1}$ in Shrestha et al.'s experimental work. ${ }^{13}$ The reason is that the PE's Rouse relaxation time here is much shorter than those in experiments due to the lower molecular weight used here. Therefore, high strain rates are required to match the short Rouse relaxation time here to yield a suitable Deborah number (relaxation time $x$ strain rates) that can achieve the optimal morphology and TC of PE fibers. Uniaxial deformation was applied along the $z$-axis of the simulation box, until reaching an engineering strain of 20 . The pressures in the lateral dimensions were kept constant at 1 bar during the deformation. When the desired strain value was achieved, each strained sample was quenched to $300 \mathrm{~K}$ instantly and stress relaxed under the NPT ensemble $(P=1 \mathrm{bar}$ and $T=300 \mathrm{~K})$ for $20 \mathrm{~ns}$ to release all the residue stresses, mimicking the status of post-processed PE fibers. Figure $1 \mathrm{~b}$ shows the simulation snapshots of the PE fiber morphology under different strain levels after the quenching and the stress relaxation processes at $300 \mathrm{~K}$. Figure $1 \mathrm{c}$ depicts the flowchart of the simulated fiber thermal-drawing process.

\section{Stress relaxation modulus calculations}

The stress relaxation modulus $G(t)$ for undrawn bulk amorphous PE were measured using EMD simulations through the stress autocorrelation 
function:

$$
G(t)=\frac{V}{k_{\mathrm{B}} T}\left\langle\sigma_{a b}(0) \sigma_{a b}(t)\right\rangle
$$

where $k_{\mathrm{B}}$ is the Boltzmann constant, $\sigma_{a b}$ are the off-diagonal $x z$ and $y z$ shear components of the stress tensor, and $z$ is the fiber-drawing direction. In EMD simulations, the system was first relaxed under the NPT ensemble at 1 bar (anisotropic pressure coupling, where each dimension is able to change) and corresponding temperatures (300,400,500, and $600 \mathrm{~K}$ ) to remove the residual stresses. Then, the shear stresses were collected every $20 \mathrm{fs}$ over a long period of $1 \mu \mathrm{s}$ under the $N V T$ ensemble to calculate $G(t)$ using the multiple-tau correlator method. ${ }^{52}$

\section{TC calculations}

The TCs of single PE chain and bulk PE system were calculated using the NEMD simulations through the Müller-Plathe algorithm. ${ }^{53} \mathrm{~A}$ schematic of the simulation system used to compute the TCs of bulk PE systems is shown in the inset of Supplementary Fig. 16b. In NEMD, we induce a heat flux $J_{\text {total }}$ by exchanging the velocity of the hottest atom in the cold slab with that of the coldest atom in the hot slab. For the systems to reach steady states in which the temperature profile is stable, it typically takes $1 \mathrm{~ns}$ for AA-MD simulations of single PE chains, and $20 \mathrm{~ns}$ for CG-MD simulations of both single PE chains and bulk PE systems. After reaching steady states, the temperature of each slab was averaged over another $1 \mathrm{~ns}$ for AA-MD simulations and $60 \mathrm{~ns}$ for CG-MD simulations. Supplementary Fig. 16b shows a typical temperature profile of the simulation system along the $z$-axis. The temperature gradient $\partial T / \partial z$ was then obtained by fitting the linear regions of the temperature profile using the least-squares method. Note that the artificial selection of linear regions could result in deviations in the computed TC. For a single PE chain, the deviation is quite small (see Supplementary Fig. 12), while the deviation for bulk PE systems could be negligible due to the highly linear temperature profile obtained from the NEMD simulations (see Supplementary Fig. 16b). Sequentially, the total TC can be computed from the Fourier's law:

$\kappa_{\text {total }}=-\frac{J_{\text {total }}}{(\partial T / \partial z) A}$,

where $A$ is the cross-sectional area for heat flux. For single PE chain systems, $A=18 \AA^{2}$, based on the unit cell dimensions of a single chain in an idealized bulk PE crystal structure. ${ }^{9}$ For bulk PE systems, $A$ equals to the cross-sectional area of the simulation box. All TC calculations were performed at $300 \mathrm{~K}$ using NEMD simulations.

TC contributions from various mechanisms are revealed by decomposing the total heat flux in NEMD simulations, which is given by the following equation: ${ }^{54,55}$

$J_{\text {total }}=\frac{1}{V}\left(\sum_{i} e_{i} v_{i}-\sum_{i} s_{i} v_{i}\right)$,

where $e_{i}$ and $v_{i}$ are the internal (potential and kinetic) energy and velocity of bead $i$, respectively. $S_{i}$ for bead $i$ is calculated using the following equation:

$$
S_{a b}=-\left[\begin{array}{c}
\frac{1}{2} \sum_{n=1}^{N_{\mathrm{p}}}\left(r_{1 a} F_{1 b}+r_{2 a} F_{2 b}\right)+\frac{1}{2} \sum_{n=1}^{N_{b}}\left(r_{1 a} F_{1 b}+r_{2 a} F_{2 b}\right) \\
+\frac{1}{3} \sum_{n=1}^{N_{a}}\left(r_{1 a} F_{1 b}+r_{2 a} F_{2 b}+r_{3 a} F_{3 b}\right)
\end{array}\right],
$$

where $S_{a b}$ is the $a b$ components of $S_{i} ; r_{1}, r_{2}$, and $r_{3}$ are positions of interacting beads 1,2 , and 3 , respectively; $F_{1}, F_{2}$, and $F_{3}$ are the forces acting on beads 1,2 , and 3 , respectively; and $N_{\mathrm{p}}, N_{\mathrm{b}}$, and $N_{\mathrm{a}}$ are the numbers of nonbonded ( $\mathrm{vdW}$ here), covalent bonded and angular interactions acting on bead $i$, respectively. The first and second terms on the right of Eq. 5 are the convection and virial contributions due to different interactions, respectively. Therefore, the total heat flux, $J_{\text {total, }}$ is decomposed into contributions from convection and virial contributions due to different interactions:

$J_{\text {total }}=J_{\text {convection }}+J_{\text {nonbonded }}+J_{\text {bond }}+J_{\text {angle }}$

The total TC, $\kappa_{\text {total, }}$ is then decomposed into contributions from convection, nonbonded, bonded, and angular interactions by the corresponding heat flux term using the Fourier's law. ${ }^{56}$

To calculate TCs of perfect PE crystals composed of chains with finite length, we need to consider the interfacial thermal resistance when two chains are joint head-to-tail via nonbonded interactions. As a result, we expect discontinuities in the temperature profile for a PE crystal, if we calculated TC using the Müller-Plathe algorithm. Therefore, the TCs of perfect $P E$ crystals at $300 \mathrm{~K}$ were determined using the EMD simulation via the Green-Kubo method. ${ }^{47}$ In EMD, after energy minimization, the system was relaxed under the NPT ensemble with constant pressure of $1 \mathrm{bar}$ for $20 \mathrm{~ns}$, followed by an extensive sampling for $1.6 \mu \mathrm{s}$ to integrate the heat flux autocorrelation function for computing $\kappa_{i}$ along dimension $i(x, y$ or $z$ ). Specifically,

$\kappa_{i}=\frac{V}{k_{\mathrm{B}} T^{2}} \int_{0}^{\infty}\left\langle J_{i}(0) J_{i}(t)\right\rangle \mathrm{d} t$,

where $J_{i}$ is the heat flux along the ith dimension.

Orientational order parameter and degree of crystallinity

Orientational order parameter $22,57,58$ is a very useful measure for the alignment of polymer chains along the applied strain direction. The local chain orientation at each bead $i$ can be computed by the chord vectors connected to the bead: $\mathbf{e}_{i}=\left(\mathbf{r}_{i+1}-\mathbf{r}_{i-1}\right) /\left|\mathbf{r}_{i+1}-\mathbf{r}_{i-1}\right|$. The total orientational order parameter $P_{2 z}$ along the $z$ direction was calculated as:

$P_{2 z}=\frac{\sum_{i=1}^{N} 3\left\langle\left(\mathbf{e}_{i} \cdot \mathbf{e}_{z}\right)^{2}\right\rangle-1}{2 N}$,

where $\mathbf{e}_{z}$ is the unit vector along the direction of the applied strain and $N$ is the total number of beads.

To characterize each bead $i$ as either crystalline or amorphous, the local orientational order parameter $P_{2, i}{ }^{59}$ also a measure of local degree of crystallinity, is calculated as:

$P_{2, i}=\frac{\sum_{j=1}^{n} 3\left\langle\left(\mathbf{e}_{i} \cdot \mathbf{e}_{j}\right)^{2}\right\rangle-1}{2 n}$,

where $\mathbf{e}_{j}$ is the unit chord vector at bead $j$ and $n$ is the number of beads $j$ that are within a cutoff distance of $10 \AA$ from bead $i$. If $P_{2, i}$ is larger than a threshold value of 0.7 , bead $i$ is regarded as a crystalline bead, otherwise it is regarded as an amorphous bead. Then, the degree of crystallinity $\psi$ of the system is calculated as $\psi=N_{\mathrm{C}} / N$, where $N_{\mathrm{c}}$ is the number of crystalline beads. We also estimated $\psi$ by the density method used in regular experiments. Specifically, for a PE system with an averaged density $\rho$, the degree of crystallinity can be defined as $\psi=\frac{\rho-\rho_{0}}{\rho_{\text {cry }}-\rho_{0}}$, where $\rho_{0}$ and $\rho_{\text {cry }}$ are the densities of amorphous and perfect crystalline PE systems, respectively. The $\psi$ values determined by the density method are lower than those by the order parameter method in this work, but they share the same trend with increasing the strain (see Supplementary Fig. 13). Therefore, we expect that the differences derived from these two methods would not change the main conclusions in this work.

\section{Intrinsic phonon property calculations}

The velocity autocorrelation function (VACF) averaged over all CG beads was computed from $40 \mathrm{ps}$ of CG-MD simulation under the NPT $(P=1 \mathrm{bar}$ and $T=300 \mathrm{~K})$ ensemble with velocities sampled every $20 \mathrm{fs}$. The normalized phonon VDOS is obtained by taking the discrete Fourier transform of the VACF. Specifically, the VDOS, $D(\omega)$, can be expressed by

$D(\omega)=\frac{1}{2 \pi}\left[\int_{0}^{\infty} \frac{\langle v(0) v(t)\rangle}{\langle v(0) v(0)\rangle} \exp (-\mathrm{i} \omega t) \mathrm{d} t\right]^{2}$,

where $\omega$ is the phonon angular frequency, and $v$ is the velocity of each CG bead.

According to the kinetic theory of phonon transport; ${ }^{60}$ in general, the TC $K$ is proportional to the effective phonon MFP $l_{\text {eff }}$ through:

$\kappa \sim \sum_{i} C_{i} v_{i} l_{i}=l_{\mathrm{eff}} \sum_{i} C_{i} v_{i}$,

where $C_{i}, v_{i}$, and $I_{i}$ denote the phonon specific heat, group velocity, and MFP of mode-i phonon, respectively. For a fully condensed bulk polymer system without voids/vacancies, the intrinsic Umklapp phonon-phonon scattering and the phonon-boundary scattering are considered as the primary phonon scattering mechanisms in NEMD simulations. Assuming that phonons scatter through the Matthiessen's rule based on the phonon relaxation time approximation, ${ }^{61-63}$ the $\kappa$ value can be correlated to 
different phonon MFPs as:

$\frac{1}{\kappa} \sim \frac{1}{l_{\mathrm{eff}}}=\frac{1}{l_{\mathrm{ph}-\mathrm{ph}}}+\frac{1}{l_{\mathrm{ph}-\mathrm{b}}}$,

where $I_{\text {ph-ph }}$ is the intrinsic phonon MFP due to three-phonon Umklapp scattering and $I_{\text {ph-b }}$ is the phonon MFP due to phonon-boundary scattering. $I_{\mathrm{ph}-\mathrm{b}}$ can be estimated by the half lengths of the simulation cell along the $z$-axis here due to the extra heat source and sink introduced in the Müller-Plathe algorithm. ${ }^{53}$ To study the intrinsic phonon-phonon scattering and estimate TCs for macroscopic polymer fibers, samples at strains of $0,2,4,6,12$, and 18 were replicated two and four times along the strain direction (z-axis) to obtain different cell lengths. For these simulation cells at different lengths, the NEMD simulations were performed for $80-800 \mathrm{~ns}$ depending on the size of the system in order to obtain converged temperature profile and TC. The intrinsic MFP $I_{\mathrm{ph}-\mathrm{ph}}$ was then evaluated by linearly fitting the $1 / \kappa(L)$ vs. $1 / /_{\text {ph-b }}$ curve (Supplementary Fig. 11).

The primary phonon group velocities can be estimated by the elastic moduli of the system using the following equation: $:^{0}$

$v_{\mathrm{L}} \approx \sqrt{E / \rho}$ and $v_{\mathrm{T}} \approx \sqrt{G / \rho}$,

where $v_{\mathrm{L}}$ and $v_{\mathrm{T}}$ are the longitudinal and transverse group velocities, respectively; $E$ and $G$ are the Young's modulus and the shear modulus, respectively; and $\rho$ is the mass density. CG-MD tensile and shear deformation simulations were both conducted to obtain the stress-strain curves, from which the elastic moduli $E$ and $G$ (see Supplementary Fig. 14) could be estimated by linearly fitting the linear elastic region. Note that the stress-strain curves were obtained from post-drawn samples (after stress relaxation and quenched to $300 \mathrm{~K}$ ) collected under various strain levels; therefore, we can obtain strain-dependent elastic moduli and the corresponding primary group velocities.

\section{DATA AVAILABILITY}

All data generated/analyzed during the current study are available from the corresponding author upon reasonable request.

\section{CODE AVAILABILITY}

All codes written to generate/analyze the data during the current study are available from the corresponding author upon reasonable request.

Received: 18 July 2019; Accepted: 2 December 2019; Published online: 19 December 2019

\section{REFERENCES}

1. Cahilla, D. G. et al. Nanoscale thermal transport. J. Appl. Phys. 93, 793-818 (2003)

2. Bock, K. Polymer electronics systems-polytronics. Proc. IEEE 93, 1400-1406 (2005).

3. Baur, J. \& Silverman, E. Challenges and opportunities in multifunctional nanocomposite structures for aerospace applications. MRS Bull. 32, 328-334 (2007).

4. Holbery, J. \& Houston, D. Natural-fiber-reinforced polymer composites in automotive applications. JOM 58, 80-86 (2006).

5. Burger, N. et al. Review of thermal conductivity in composites: mechanisms, parameters and theory. Prog. Polym. Sci. 61, 1-28 (2016).

6. Zhong, Z. et al. Structure-induced enhancement of thermal conductivities in electrospun polymer nanofibers. Nanoscale 6, 8283-8291 (2014).

7. Chae, H. G. \& Kumar, S. Making strong fibers. Science 319, $908-909$ (2008).

8. Zhou, X. W. \& Jones, R. E. Effects of nano-void density, size and spatial population on thermal conductivity: a case study of GaN crystal. J. Phys. 24, 325801-325815 (2012).

9. Henry, A. \& Chen, G. High thermal conductivity of single polyethylene chains using molecular dynamics simulations. Phys. Rev. Lett. 101, 235502 (2008).

10. Shen, S., Henry, A., Tong, J., Zheng, R. \& Chen, G. Polyethylene nanofibres with very high thermal conductivities. Nat. Nanotechnol. 5, 251-255 (2010).

11. Choy, C. L., Wong, Y. W., Yang, G. W. \& Kanamoto, T. Elastic modulus and thermal conductivity of ultradrawn polyethylene. J. Polym. Sci. Part B 37, 3359-3367 (1999).

12. Zhu, B. et al. Novel polyethylene fibers of very high thermal conductivity enabled by amorphous restructuring. ACS Omega 2, 3931-3944 (2017).

13. Shrestha, R. et al. Crystalline polymer nanofibers with ultra-high strength and thermal conductivity. Nat. Commun. 9, 1664 (2018).
14. Liao, Q., Liu, Z., Liu, W., Deng, C. \& Yang, N. Extremely high thermal conductivity of aligned carbon nanotube-polyethylene composites. Sci. Rep. 5, 16543 (2015).

15. Vadukumpully, S., Paul, J., Mahanta, N. \& Valiyaveettil, S. Flexible conductive graphene/poly (vinyl chloride) composite thin films with high mechanical strength and thermal stability. Carbon 49, 198-205 (2011).

16. Zhang, T., Wu, X. \& Luo, T. Polymer nanofibers with outstanding thermal conductivity and thermal stability: fundamental linkage between molecular characteristics and macroscopic thermal properties. J. Phys. Chem. C 118, 21148-21159 (2014).

17. Tu, R., Liao, Q., Zeng, L., Liu, Z. \& Liu, W. Impact of torsion and stretching on the thermal conductivity of polyethylene strands. Appl. Phys. Lett. 110, 101905 (2017).

18. Ma, H. \& Tian, Z. Effects of polymer chain confinement on thermal conductivity of ultrathin amorphous polystyrene films. Appl. Phys. Lett. 107, 073111 (2015).

19. Li, S., Yu, X., Bao, H. \& Yang, N. High thermal conductivity of bulk epoxy resin by bottom-up parallel-linking and strain: a molecular dynamics study. J. Phys. Chem. C 122, 13140-13147 (2018)

20. Wei, X., Zhang, T. \& Luo, T. Chain conformation-dependent thermal conductivity of amorphous polymer blends: the impact of inter- and intra-chain interactions. Phys. Chem. Chem. Phys. 18, 32146-32154 (2016).

21. He, J., Kim, K., Wang, Y. \& Liu, J. Strain effects on the anisotropic thermal transport in crystalline polyethylene. Appl. Phys. Lett. 112, 051907 (2018).

22. Liu, J. \& Yang, R. Tuning the thermal conductivity of polymers with mechanical strains. Phys. Rev. B 81, 174122 (2010).

23. Zhang, T. \& Luo, T. High-contrast, reversible thermal conductivity regulation utilizing the phase transition of polyethylene nanofibers. ACS Nano 7, 7592-7600 (2013).

24. Ronca, S., Igarashi, T., Forte, G. \& Rastogi, S. Metallic-like thermal conductivity in a lightweight insulator: solid-state processed ultra high molecular weight polyethylene tapes and films. Polymer 123, 203-210 (2017).

25. Zhang, T. \& Luo, T. Role of morphology and stiffness in thermal conductivity of amorphous polymers. J. Phys. Chem. B 120, 803-812 (2016).

26. Mark, J. E. Physical Properties of Polymers Handbook, Vol. 1076 (Springer, 2007).

27. Liu, J. \& Yang, R. Length-dependent thermal conductivity of single extended polymer chains. Phys. Rev. B 86, 104307 (2012).

28. Zhao, J., Jiang, J.-W., Wei, N., Zhang, Y. \& Rabczuk, T. Thermal conductivity dependence on chain length in amorphous polymers. J. Appl. Phys. 113, 184304 (2013).

29. Hansen, D. \& Ho, C. C. Thermal conductivity of high polymers. J. Polym. Sci. Part A 3, 659-670 (1965).

30. Hansen, D., Kantayya, R. C. \& Ho, C. C. Thermal conductivity of high polymersthe influence of molecular weight. Polym. Eng. Sci. 6, 260-262 (1966).

31. Mehra, N. et al. Thermal transport in polymeric materials and across composite interfaces. Appl. Mater. Today 12, 92-130 (2018).

32. Marrink, S. J., Risselada, H. J., Yefimov, S., Tieleman, D. P. \& de Vries, A. H. The MARTINI force field: coarse grained model for biomolecular simulations. J. Phys. Chem. B 111, 7812-7824 (2007).

33. Lin, S. et al. Predictive modelling-based design and experiments for synthesis and spinning of bioinspired silk fibres. Nat. Commun. 6, 6892 (2015).

34. Lin, S., Zhang, J., Strano, M. S. \& Blankschtein, D. Understanding selective molecular recognition in integrated carbon nanotube-polymer sensors by simulating physical analyte binding on carbon nanotube-polymer scaffolds. Soft Matter 10, 5991-6004 (2014).

35. Zhai, C., Zhou, H., Gao, T., Zhao, L. \& Lin, S. Electrostatically tuned microdomain morphology and phase-dependent ion transport anisotropy in single-ion conducting block copolyelectrolytes. Macromolecules 51, 4471-4483 (2018).

36. Moore, T. C., lacovella, C. R. \& McCabe, C. Derivation of coarse-grained potentials via multistate iterative Boltzmann inversion. J. Chem. Phys. 140, 06B606_601 (2014).

37. Rubinstein, M. \& Colby, R. H. Polymer Physics (Oxford University Press, 2003).

38. Chellamuthu, M., Arora, D., Winter, H. \& Rothstein, J. P. Extensional flow-induced crystallization of isotactic poly-1-butene using a filament stretching rheometer. J. Rheol. 55, 901-920 (2011).

39. Choy, C. L. \& Young, K. Thermal conductivity of semicrystalline polymers-a model. Polymer 18, 769-776 (1977).

40. Fetters, L. J., Lohse, D. J. \& Graessley, W. W. Chain dimensions and entanglement spacings in dense macromolecular systems. J. Polym. Sci. Part B 37, 1023-1033 (1999).

41. Likhtman, A. E., Sukumaran, S. K. \& Ramirez, J. Linear viscoelasticity from molecular dynamics simulation of entangled polymers. Macromolecules 40, 6748-6757 (2007).

42. Reiner, M. The deborah number. Phys. Today 17, 62 (1964).

43. Chen, $\mathrm{H}$. et al. Thermal conductivity of polymer-based composites: fundamentals and applications. Prog. Polym. Sci. 59, 41-85 (2016).

44. Hennig, J. Anisotropy and structure in uniaxially stretched amorphous high polymers. J. Polym. Sci. Part C 16, 2751-2761 (1967). 
45. Ward, I. M. \& Sweeney, J. Mechanical Properties of Solid Polymers (Wiley, 2012).

46. Lu, T. et al. Thermal transport in semicrystalline polyethylene by molecular dynamics simulation. J. Appl. Phys. 123, 015107 (2018).

47. Wang, M. \& Lin, S. Ballistic thermal transport in carbyne and cumulene with micron-scale spectral acoustic phonon mean free path. Sci. Rep. 5, 18122 (2015).

48. Hands, D., Lane, K. \& Sheldon, R. P. Thermal conductivities of amorphous polymers. J. Polym. Sci. 42, 717-726 (1973).

49. Plimpton, S. Fast parallel algorithms for short-range molecular dynamics. J. Comput. Phys. 117, 1-19 (1995).

50. Stuart, S. J., Tutein, A. B. \& Harrison, J. A. A reactive potential for hydrocarbons with intermolecular interactions. J. Chem. Phys. 112, 6472-6486 (2000).

51. Auhl, R., Everaers, R., Grest, G. S., Kremer, K. \& Plimpton, S. J. Equilibration of long chain polymer melts in computer simulations. J. Chem. Phys. 119, 12718-12728 (2003).

52. Ramírez, J., Sukumaran, S. K., Vorselaars, B. \& Likhtman, A. E. Efficient on the fly calculation of time correlation functions in computer simulations. J. Chem. Phys. 133, 154103 (2010).

53. Müller-Plathe, F. A simple nonequilibrium molecular dynamics method for calculating the thermal conductivity. J. Chem. Phys. 106, 6082-6085 (1997).

54. Irving, J. H. \& Kirwood, J. G. The statistical mechanical theory of transport processes. IV. The equations of hydrodynamics. J. Chem. Phys. 18, 817-829 (1950).

55. Torii, D., Nakano, T. \& Ohara, T. Contribution of inter- and intramolecular energy transfers to heat conduction in liquids. J. Chem. Phys. 128, 044504 (2008).

56. Ohara, T., Yuan, T. C., Torii, D., Kikugawa, G. \& Kosugi, N. Heat conduction in chain polymer liquids: molecular dynamics study on the contributions of inter- and intramolecular energy transfer. J. Chem. Phys. 135, 034507 (2011).

57. Yong, C. W. \& Higgs, P. G. Chain orientation in polymer networks: computer simulations using the bond fluctuation model. Macromolecules 32, 5062-5071 (1999).

58. Gestoso, P. \& Brisson, J. Simulation of orientation of uniaxially stretched poly (vinyl phenol) by molecular dynamics. J. Polym. Sci. Part B 40, 1601-1625 (2002).

59. Yeh, I.-C., Lenhart, J. L., Rutledge, G. C. \& Andzelm, J. W. Molecular dynamics simulation of the effects of layer thickness and chain tilt on tensile deformation mechanisms of semicrystalline polyethylene. Macromolecules 50, 1700-1712 (2017).

60. Zhao, L., Xu, S., Wang, M. \& Lin, S. Probing the thermodynamic stability and phonon transport in two-dimensional hexagonal aluminum nitride monolayer. $J$. Phys. Chem. C 120, 27675-27681 (2016).

61. Holland, M. G. Analysis of lattice thermal conductivity. Phys. Rev. 132, 2461-2471 (1963).

62. Holland, M. G. Phonon scattering in semiconductors from thermal conductivity studies. Phys. Rev. 134, A471-A480 (1964).

63. Dames, C. \& Chen, G. Theoretical phonon thermal conductivity of Si/Ge superlattice nanowires. J. Appl. Phys. 95, 682-693 (2004).

\section{ACKNOWLEDGEMENTS}

We would like to acknowledge the startup grant from Shanghai Jiao Tong University and the donors of the American Chemical Society (ACS) Petroleum Research Fund (PRF) under the Doctoral New Investigator (DNI) Award \# 56499-DNI7.

\section{AUTHOR CONTRIBUTIONS}

S.L. and Z.C. contributed equally to this work. The manuscript was written through contributions of all authors. All authors have given approval to the final version of the manuscript.

\section{COMPETING INTERESTS}

The authors declare no competing interests.

\section{ADDITIONAL INFORMATION}

Supplementary information is available for this paper at https://doi.org/10.1038/ s41524-019-0264-2.

Correspondence and requests for materials should be addressed to S.L.

Reprints and permission information is available at http://www.nature.com/ reprints

Publisher's note Springer Nature remains neutral with regard to jurisdictional claims in published maps and institutional affiliations.

(c) (i)

Open Access This article is licensed under a Creative Commons Attribution 4.0 International License, which permits use, sharing, adaptation, distribution and reproduction in any medium or format, as long as you give appropriate credit to the original author(s) and the source, provide a link to the Creative Commons license, and indicate if changes were made. The images or other third party material in this article are included in the article's Creative Commons license, unless indicated otherwise in a credit line to the material. If material is not included in the article's Creative Commons license and your intended use is not permitted by statutory regulation or exceeds the permitted use, you will need to obtain permission directly from the copyright holder. To view a copy of this license, visit http://creativecommons. org/licenses/by/4.0/.

(c) The Author(s) 2019 